\title{
Further Inequalities Associated with the Classical Gamma Function
}

\author{
Kwara Nantomah* \\ Department of Mathematics, University for Development Studies, Navrongo Campus, Navrongo UE/R, Ghana \\ *Corresponding author: mykwarasoft@yahoo.com, knantomah@uds.edu.gh
}

Received December 24, 2014; Revised August 28, 2015; Accepted September 23, 2015

\begin{abstract}
In this paper, the authors present some double inequalities associated with certain ratios of the Gamma function. The results are further generalizations of several previous results. The approach is based on some monotonicity properties of some functions involving the generalized Gamma functions. At the end, some open problems are posed.
\end{abstract}

Keywords: Gamma function, Psi function, inequality, generalization

Cite This Article: Kwara Nantomah, "Further Inequalities Associated with the Classical Gamma Function.” Turkish Journal of Analysis and Number Theory, vol. 3, no. 4 (2015): 111-115. doi: 10.12691/tjant-3-4-5.

\section{Introduction}

Inequalities involving the classical Euler's Gamma function has gained the attention of researchers all over the world. Recent advances in this area include those inequalities involving ratios of the Gamma function. In $[1,5,6,10]$ and [11-17], the authors established some interesting inequalities concerning such ratios, as well as some generalizations. By utilizing similar techniques, this paper seeks to present some new results generalizing the results of [11-17]. At the end, we pose some open problems involving the generalized Psi functions. In the sequel, we recall some basic definitions concerning the Gamma function and its generalizations. These definitions are required in order to establish our results.

The well-known classical Gamma function, $\Gamma(t)$ and the classical Psi or Digamma function $\psi(t)$ are usually defined for $t>0$ as:

$$
\Gamma(t)=\int_{0}^{\infty} e^{-x} x^{t-1} d x \text { and } \psi(t)=\frac{\Gamma^{\prime}(t)}{\Gamma(t)} .
$$

The p-Gamma function, $\Gamma_{p}(t)$ and the p-Psi function $\psi_{p}(t)$ are defined for $p \in N$ and $t>0$ as:

$$
\Gamma_{p}(t)=\frac{p ! p^{t}}{t(t+1) \ldots(t+p)} \text { and } \psi_{p}(t)=\frac{\Gamma_{p}^{\prime}(t)}{\Gamma_{p}(t)}
$$

where $\Gamma_{p}(t) \rightarrow \Gamma(t)$ and $\psi_{p}(t) \rightarrow \psi(t)$ as $p \rightarrow \infty$. For more information on this function, see [9] and the references therein.

Also, the q-Gamma function, $\Gamma_{q}(t)$ and the q-Psi function $\psi_{q}(t)$ are defined for $q \in(0,1)$ and $t>0$ as:

$$
\Gamma_{q}(t)=(1-q)^{1-t} \prod_{n=1}^{\infty} \frac{1-q^{n}}{1-q^{t+n}} \text { and } \psi_{q}(t)=\frac{\Gamma_{q}^{\prime}(t)}{\Gamma_{q}(t)}
$$

where $\Gamma_{q}(t) \rightarrow \Gamma(t)$ and $\psi_{q}(t) \rightarrow \psi(t)$ as $q \rightarrow 1^{-}$.

See also $[4,5]$ and the references therein.

Similarly, the k-Gamma function, $\Gamma_{k}(t)$ and the k-Psi function $\psi_{k}(t)$ are defined for $k>0$ and $t>0$ as (see $[2,7])$ :

$$
\Gamma_{k}(t)=\int_{0}^{\infty} e^{-\frac{x^{k}}{k}} x^{t-1} d x \text { and } \psi_{k}(t)=\frac{\Gamma_{k}^{\prime}(t)}{\Gamma_{k}(t)}
$$

where $\Gamma_{k}(t) \rightarrow \Gamma(t)$ and $\psi_{k}(t) \rightarrow \psi(t)$ as $k \rightarrow 1$.

Also, the (q,k)-Gamma function $\Gamma_{(q, k)}(t)$ and the (q,k)-Psi function $\psi_{(q, k)}(t)$ are defined for $q \in(0,1)$, $k>0$ and $t>0$ as [3]:

$$
\Gamma_{(q, k)}(t)=\frac{\left(1-q^{k}\right)_{q, k}^{\frac{t}{k}-1}}{(1-q)^{\frac{t}{k}-1}} \text { and } \psi_{(q, k)}(t)=\frac{\Gamma_{(q, k)}^{\prime}(t)}{\Gamma_{(q, k)}(t)}
$$

where $(t)_{n, k}=\prod_{j=0}^{n-1}(t+j k)$ is the k-generalized Pochhammer symbol and $\Gamma_{(q, k)}(t) \rightarrow \Gamma(t), \psi_{(q, k)}(t) \rightarrow \psi(t)$ as $q \rightarrow 1^{-}$, $k \rightarrow 1$.

Furthermore, the (p,q)-Gamma function $\Gamma_{(p, q)}(t)$ and the $(\mathrm{p}, \mathrm{q})$-Psi function $\psi_{(p, q)}(t)$ are defined for $p \in N$, $q \in(0,1)$ and $t>0$ as [8]:

$$
\Gamma_{(p, q)}(t)=\frac{[p]_{q}^{t}[p]_{q} !}{[t]_{q}[t+1]_{q} \cdots[t+p]_{q}}
$$


and

$$
\psi_{(p, q)}(t)=\frac{\Gamma_{(p, q)}^{\prime}(t)}{\Gamma_{(p, q)}(t)}
$$

where $[p]_{q}=\frac{1-q^{p}}{1-q}$, and $\Gamma_{(p, q)}(t) \rightarrow \Gamma(t), \psi_{(p, q)}(t) \rightarrow \psi(t)$ as $p \rightarrow \infty, q \rightarrow 1^{-}$.

As defined above, the generalized Psi functions: $\psi_{p}(t)$, $\psi_{q}(t), \psi_{k}(t), \psi_{(q, k)}(t)$ and $\psi_{(p, q)}(t)$ possess the following series forms (see $[16,17]$ and the references therein):

$$
\begin{gathered}
\psi_{p}(t)=\operatorname{In} p-\sum_{n=0}^{p} \frac{1}{n+t} \\
\psi_{q}(t)=-\operatorname{In}(1-q)+(\operatorname{In} q) \sum_{n=1}^{\infty} \frac{q^{n t}}{1-q^{n}} \\
\psi_{k}(t)=\frac{\operatorname{In} k-\gamma}{k}-\frac{1}{t}+\sum_{n=1}^{\infty} \frac{t}{n k(n k+t)} \\
\psi_{(p, q)}(t)=\operatorname{In}[p]_{q}+(\operatorname{In} q) \sum_{n=1}^{p} \frac{q^{n t}}{1-q^{n}} \\
\psi_{(q, k)}(t)=\frac{-\operatorname{In}(1-q)}{k}+(\operatorname{In} q) \sum_{n=1}^{\infty} \frac{q^{n k t}}{1-q^{n k}}
\end{gathered}
$$

with $\gamma=\lim _{n \rightarrow \infty}\left(\sum_{k=1}^{n} \frac{1}{k}-\operatorname{Inn}\right)=0.5721566 \ldots$ denoting the Euler-Mascheroni’s constant.

\section{Results}

We now present our results. Let us begin with the following Lemmas pertaining to the results.

Lemma 2.1. Assume that $\lambda \geq \mu>0, p \in N, q \in(0,1)$ and $g(t)>0$. Then,

$$
\begin{aligned}
& \lambda \operatorname{In}(1-q)+\mu \operatorname{In}[p]_{q} \\
& +\lambda \psi_{q}(g(t))-\mu \psi_{(p, q)}(g(t)) \leq 0 .
\end{aligned}
$$

Proof. By using equations (2) and (4) we obtain,

$$
\begin{aligned}
& \lambda \operatorname{In}(1-q)+\mu \operatorname{In}[p]_{q}+\lambda \psi_{q}(g(t))-\mu \psi_{(p, q)}(g(t)) \\
= & (\operatorname{In} q)\left[\lambda \sum_{n=1}^{\infty} \frac{q^{n g(t)}}{1-q^{n}}-\mu \sum_{n=1}^{p} \frac{q^{n g(t)}}{1-q^{n}}\right] \leq 0 .
\end{aligned}
$$

concluding the proof.

Lemma 2.2. Assume that $\lambda \geq \mu>0, q \in(0,1), k \geq 1$ and $g(t)>0$. Then,

$$
\begin{aligned}
& \lambda \operatorname{In}(1-q)-\mu \frac{\operatorname{In}(1-q)}{k} \\
& +\lambda \psi_{q}(g(t))-\mu \psi_{(q, k)}(g(t)) \leq 0 .
\end{aligned}
$$

Proof. By using equations (2) and (5) we obtain,

$$
\begin{aligned}
& \lambda \operatorname{In}(1-q)-\mu \frac{\operatorname{In}(1-q)}{k} \\
+ & \lambda \psi_{q}(g(t))-\mu \psi_{(q, k)}(g(t)) \\
= & (\operatorname{In} q) \sum_{n=1}^{\infty}\left[\lambda \frac{q^{n g(t)}}{1-q^{n}}-\mu \frac{q^{n k g(t)}}{1-q^{n k}}\right] \leq 0
\end{aligned}
$$

concluding the proof.

Lemma 2.3. Assume that $\lambda>0, \mu>0, k>0, p \in N$, $q \in(0,1)$ and $g(t)>0$. Then,

$$
\begin{aligned}
& \mu \operatorname{In}[p]_{q}-\frac{\lambda \operatorname{In} k}{k}+\frac{\lambda \gamma}{k}+\frac{\lambda}{g(t)} \\
& +\lambda \psi_{k}(g(t))-\mu \psi_{(p, q)}(g(t))>0 .
\end{aligned}
$$

Proof. By using equations (3) and (4) we obtain,

$$
\begin{aligned}
& \mu \operatorname{In}[p]_{q}-\frac{\lambda \operatorname{Ink}}{k}+\frac{\lambda \gamma}{k}+\frac{\lambda}{g(t)} \\
& +\lambda \psi_{k}(g(t))-\mu \psi_{(p, q)}(g(t)) \\
= & \lambda \sum_{n=1}^{\infty} \frac{g(t)}{n k(n k+g(t))}-\mu(\operatorname{In} q) \sum_{n=1}^{p} \frac{q^{n g(t)}}{1-q^{n}}>0
\end{aligned}
$$

concluding the proof.

Lemma 2.4. Assume that $\lambda>0, \mu>0, q \in(0,1)$, $k>0$ and $g(t)>0$. Then,

$$
\begin{aligned}
& \frac{\lambda \gamma}{k}+\frac{\lambda}{g(t)}-\frac{\operatorname{In}\left(k^{\lambda}(1-q)^{\mu}\right)}{k} \\
& +\lambda \psi_{k}(g(t))-\mu \psi_{(q, k)}(g(t))>0 .
\end{aligned}
$$

Proof. By using equations (3) and (5) we obtain,

$$
\begin{aligned}
& \frac{\lambda \gamma}{k}+\frac{\lambda}{g(t)}-\frac{\operatorname{In}\left(k^{\lambda}(1-q)^{\mu}\right)}{k} \\
& +\lambda \psi_{k}(g(t))-\mu \psi_{(q, k)}(g(t)) \\
& =\lambda \sum_{n=1}^{\infty} \frac{g(t)}{n k(n k+g(t))}-\mu(\operatorname{In} q) \sum_{n=1}^{\infty} \frac{q^{n k g(t)}}{1-q^{n k}}>0
\end{aligned}
$$

concluding the proof.

Theorem 2.5. Let $g(t)$ be a positive, increasing and differentiable function, $p \in N$ and $q \in(0,1)$. Then for positive real numbers $\lambda$ and $\mu$ such that $\lambda \geq \mu$, the inequalities:

$$
\begin{aligned}
& \frac{(1-q)^{\lambda(g(0)-g(x))} \Gamma_{q}(g(0))^{\lambda}}{[p]_{q}^{-\mu(g(0)-g(x))} \Gamma_{(p, q)}(g(0))^{\mu}} \\
& \geq \frac{\Gamma_{q}(g(x))^{\lambda}}{\Gamma_{(p, q)}(g(x))^{\mu}} \\
& \geq \frac{(1-q)^{\lambda(g(y)-g(x))} \Gamma_{q}(g(y))^{\lambda}}{[p]_{q}^{-\mu(g(y)-g(x))} \Gamma_{(p, q)}(g(y))^{\mu}}
\end{aligned}
$$

hold true for $0<x<y$.

Proof. Define a function $G$ for $p \in N$ and $q \in(0,1)$ by 


$$
G(t)=\frac{(1-q)^{\lambda g(t)} \Gamma_{q}(g(t))^{\lambda}}{[p]_{q}^{-\mu g(t)} \Gamma_{(p, q)}(g(t))^{\mu}}, \quad t \in(0, \infty) .
$$

Let $u(t)=\operatorname{InG}(t)$. Then,

$$
\begin{aligned}
u(t)= & \operatorname{In} \frac{(1-q)^{\lambda g(t)} \Gamma_{q}(g(t))^{\lambda}}{[p]_{q}^{-\mu g(t)} \Gamma_{(p, q)}(g(t))^{\mu}} \\
= & \lambda g(t) \operatorname{In}(1-q)+\mu g(t) \operatorname{In}[p]_{q} \\
& +\lambda \operatorname{Inn} \Gamma_{q}(g(t))-\mu \operatorname{In} \Gamma_{(p, q)}(g(t)) .
\end{aligned}
$$

Then,

$$
\begin{aligned}
u^{\prime}(t)= & \lambda g^{\prime}(t) \operatorname{In}(1-q)+\mu g^{\prime}(t) \operatorname{In}[p]_{q} \\
& +\lambda g^{\prime}(t) \psi_{q}(g(t))-\mu g^{\prime}(t) \psi_{(p, q)}(g(t)) \\
= & g^{\prime}(t)\left[\lambda \operatorname{In}(1-q)+\mu \operatorname{In}[p]_{q}\right. \\
& \left.+\lambda \psi_{q}(g(t))-\mu \psi_{(p, q)}(g(t))\right] \leq 0
\end{aligned}
$$

as a consequence of Lemma 2.1. That implies $u$ is nonincreasing on $t \in(0, \infty)$. Hence $G=e^{u(t)}$ is nonincreasing and for $0<x<y$ we have,

$$
G(0) \geq G(x) \geq G(y)
$$

establishing the inequalities in (6).

Theorem 2.6. Let $g(t)$ be a positive, increasing and differentiable function, $q \in(0,1)$ and $k \geq 1$. Then for positive real numbers $\lambda$ and $\mu$ such that $\lambda \geq \mu$, the inequalities:

$$
\begin{aligned}
& \frac{(1-q)^{\lambda(g(0)-g(x))} \Gamma_{q}(g(0))^{\lambda}}{(1-q)^{\frac{\mu}{k}(g(0)-g(x))} \Gamma_{(q, k)}(g(0))^{\mu}} \\
& \geq \frac{\Gamma_{q}(g(x))^{\lambda}}{\Gamma_{(q, k)}(g(x))^{\mu}} \\
& \geq \frac{(1-q)^{\lambda(g(y)-g(x))} \Gamma_{q}(g(y))^{\lambda}}{(1-q)^{\frac{\mu}{k}(g(y)-g(x))} \Gamma_{(q, k)}(g(y))^{\mu}}
\end{aligned}
$$

hold true for $0<x<y$.

Proof. Define a function $H$ for $q \in(0,1)$ and $k \geq 1$ by

$$
H(t)=\frac{(1-q)^{\lambda g(t)} \Gamma_{q}(g(t))^{\lambda}}{(1-q)^{\frac{\mu g(t)}{k}} \Gamma_{(q, k)}(g(t))^{\mu}}, \quad t \in(0, \infty) .
$$

Let $v(t)=\operatorname{In} H(t)$. Then,

$$
\begin{aligned}
v(t)= & \operatorname{In} \frac{(1-q)^{\lambda g(t)} \Gamma_{q}(g(t))^{\lambda}}{(1-q)^{\frac{\mu g(t)}{k}} \Gamma_{(q, k)}(g(t))^{\mu}} \\
= & \lambda g(t) \operatorname{In}(1-q)-\frac{\mu g(t)}{k} \operatorname{In}(1-q) \\
& +\lambda \operatorname{In} \Gamma_{q}(g(t))-\mu \operatorname{In} \Gamma_{(q, k)}(g(t)) .
\end{aligned}
$$

Then,

$$
\begin{aligned}
v^{\prime}(t)= & \lambda g^{\prime}(t) \operatorname{In}(1-q)-\frac{\mu g^{\prime}(t)}{k} \operatorname{In}(1-q) \\
& +\lambda g^{\prime}(t) \psi_{q}(g(t))-\mu g^{\prime}(t) \psi_{(q, k)}(g(t)) \\
= & g^{\prime}(t)\left[\lambda \operatorname{In}(1-q)+\mu \frac{\operatorname{In}(1-q)}{k}\right. \\
& \left.+\lambda \psi_{q}(g(t))-\mu \psi_{(q, k)}(g(t))\right] \leq 0
\end{aligned}
$$

as a consequence of Lemma 2.2. That implies $v$ is nonincreasing on $t \in(0, \infty)$. Hence $H=e^{v(t)}$ is nonincreasing and for $0<x<y$ we have,

$$
H(0) \geq H(x) \geq H(y)
$$

establishing the inequalities in (7).

Theorem 2.7. Let $g(t)$ be a positive, increasing and differentiable function, $k>0, p \in N$ and $q \in(0,1)$. Then for positive real numbers $\lambda$ and $\mu$, the inequalities:

$$
\begin{aligned}
& \frac{(g(0))^{\lambda} k^{-\frac{\lambda}{k}(g(0)-g(x))} e^{\frac{\lambda \gamma}{k}(g(0)-g(x))} \Gamma_{k}(g(0))^{\lambda}}{(g(x))^{\lambda}[p]_{q}^{-\mu(g(0)-g(x))} \Gamma_{(p, q)}(g(0))^{\mu}} \\
& <\frac{\Gamma_{k}(g(x))^{\lambda}}{\Gamma_{(p, q)}(g(x))^{\mu}}< \\
& \frac{(g(y))^{\lambda} k^{-\frac{\lambda}{k}(g(y)-g(x))} e^{\frac{\lambda \gamma}{k}(g(y)-g(x))} \Gamma_{k}(g(y))^{\lambda}}{(g(x))^{\lambda}[p]_{q}^{-\mu(g(y)-g(x))} \Gamma_{(p, q)}(g(y))^{\mu}}
\end{aligned}
$$

hold true for $0<x<y$.

Proof. Define a function $S$ for $k>0, p \in N$ and $q \in(0,1)$ by

$$
S(t)=\frac{(g(t))^{\lambda} k^{-\frac{\lambda g(t)}{k}} e^{\frac{\lambda \gamma g(t)}{k}} \Gamma_{k}(g(t))^{\lambda}}{[p]_{q}^{-\mu g(t)} \Gamma_{(p, q)}(g(t))^{\mu}}, t \in(0, \infty) .
$$

Let $w(t)=\operatorname{In} S(t)$. Then,

$$
\begin{aligned}
& w(t)=\operatorname{In} \frac{(g(t))^{\lambda} k^{-\frac{\lambda g(t)}{k}} e^{\frac{\lambda \gamma g(t)}{k}} \Gamma_{k}(g(t))^{\lambda}}{[p]_{q}^{-\mu g(t)} \Gamma_{(p, q)}(g(t))^{\mu}} \\
& =\mu g(t) \operatorname{In}[p]_{q}-\frac{\lambda g(t)}{k} \operatorname{In} k+\frac{\lambda \gamma g(t)}{k}+\lambda \operatorname{In}(g(t)) \\
& +\lambda \operatorname{In} \Gamma_{k}(g(t))-\mu \operatorname{In} \Gamma_{(p, q)}(g(t)) .
\end{aligned}
$$

Then,

$$
\begin{aligned}
w^{\prime}(t)= & \mu g^{\prime}(t) \operatorname{In}[p]_{q}-\frac{\lambda g^{\prime}(t) \operatorname{In} k}{k}+\frac{\lambda \gamma g^{\prime}(t)}{k} \\
+\lambda & \frac{g^{\prime}(t)}{g(t)}+\lambda g^{\prime}(t) \psi_{k}(g(t))-\mu g^{\prime}(t) \psi_{(p, q)}(g(t)) \\
= & g^{\prime}(t)\left[\mu \operatorname{In}[p]_{q}-\frac{\lambda \operatorname{In} k}{k}+\frac{\lambda \gamma}{k}+\frac{\lambda}{g(t)}\right. \\
& \left.+\lambda \psi_{k}(g(t))-\mu \psi_{(p, q)}(g(t))\right]>0
\end{aligned}
$$

as a result of Lemma 2.3. That implies $w$ is increasing on $t \in(0, \infty)$. Hence $S=e^{w(t)}$ is increasing and for $0<x<y$ we have, 


$$
S(0)<S(x)<S(y)
$$

establishing the inequalities in (8).

Theorem 2.8. Let $g(t)$ be a positive, increasing and differentiable function, $k>0$ and $q \in(0,1)$. Then for positive real numbers $\lambda$ and $\mu$, the inequalities:

$$
\begin{aligned}
& \frac{(g(x))^{-\lambda}(g(0))^{\lambda} e^{\frac{\lambda \gamma}{k}(g(0)-g(x))} \Gamma_{k}(g(0))^{\lambda}}{k^{\frac{\lambda}{k}(g(0)-g(x))}(1-q)^{\frac{\mu}{k}(g(0)-g(x))} \Gamma_{(q, k)}(g(0))^{\mu}} \\
& <\frac{\Gamma_{k}(g(x))^{\lambda}}{\Gamma_{(q, k)}(g(x))^{\mu}}< \\
& \frac{(g(x))^{-\lambda}(g(y))^{\lambda} e^{\frac{\lambda \gamma}{k}(g(y)-g(x))} \Gamma_{k}(g(y))^{\lambda}}{k^{\frac{\lambda}{k}(g(y)-g(x))}(1-q)^{\frac{\mu}{k}(g(y)-g(x))} \Gamma_{(q, k)}(g(y))^{\mu}}
\end{aligned}
$$

hold true for $0<x<y$.

Proof. Define a function $T$ for $k>0$ and $q \in(0,1)$ by

$$
T(t)=\frac{(g(t))^{\lambda} e^{\frac{\lambda \gamma g(t)}{k}} \Gamma_{k}(g(t))^{\lambda}}{k^{\frac{\lambda g(t)}{k}}(1-q)^{\frac{\mu g(t)}{k}} \Gamma_{(q, k)}(g(t))^{\mu}}, \quad t \in(0, \infty) .
$$

Let $\delta(t)=\operatorname{In} T(t)$. Then,

$$
\begin{aligned}
& \delta(t)=\operatorname{In} \frac{(g(t))^{\lambda} e^{\frac{\lambda \gamma g(t)}{k}} \Gamma_{k}(g(t))^{\lambda}}{k^{\frac{\lambda g(t)}{k}}(1-q)^{\frac{\mu g(t)}{k}} \Gamma_{(q, k)}(g(t))^{\mu}} \\
& =\lambda \operatorname{In} g(t)+\frac{\lambda \gamma g(t)}{k}-\frac{\lambda g(t)}{k} \operatorname{In} k-\frac{\mu g(t)}{k} \operatorname{In}(1-q) \\
& \quad+\lambda \operatorname{In} \Gamma_{k}(g(t))-\mu \operatorname{In} \Gamma_{(q, k)}(g(t)) .
\end{aligned}
$$

Then,

$$
\begin{aligned}
\delta^{\prime}(t)= & \frac{\lambda \gamma g^{\prime}(t)}{k}+\lambda \frac{g^{\prime}(t)}{g(t)}-\frac{g^{\prime}(t) \operatorname{In}\left(k^{\lambda}(1-q)^{\mu}\right)}{k} \\
& +\lambda g^{\prime}(t) \psi_{k}(g(t))-\mu g^{\prime}(t) \psi_{(q, k)}(g(t)) \\
= & g^{\prime}(t)\left[\frac{\lambda \gamma}{k}+\frac{\lambda}{g(t)}-\frac{\operatorname{In}\left(k^{\lambda}(1-q)^{\mu}\right)}{k}\right. \\
& \left.+\lambda \psi_{k}(g(t))-\mu \psi_{(q, k)}(g(t))\right]>0
\end{aligned}
$$

as a result of Lemma 2.4. That implies $\delta$ is -increasing on $t \in(0, \infty)$. Hence $T=e^{\delta(t)}$ is increasing and for $0<x<y$ we have,

$$
T(0)<T(x)<T(y)
$$

establishing the inequalities in (9).

\section{Concluding Remarks}

In particular, if we let $g(t)=\alpha+\beta t$ for $\alpha>0$ and $\beta>0$ on the interval $0<t<1$, then we recover the entire results of [17]. Also, by setting $g(t)=\alpha+t$ and $\lambda=\mu=1$ on the interval $0<t<1$, we obtain the results of [16]. The results [11] - [17] are therefore special cases of the results of this paper. For example, let $g(t)=\alpha+\beta t$ for $\alpha, \beta>0$ on the interval

$0<t<1$. Then;

(i) by allowing $q \rightarrow 1$ in Theorem 2.5, we recover Theorem 3.7 of [13].

(ii) by allowing $k \rightarrow 1$ in Theorem 2.8, we recover Theorem 3.8 of [13].

(iii) by allowing $q \rightarrow 1$ in Theorem 2.6, we recover Theorem 3.9 of [13].

(iv) by allowing $k \rightarrow 1$ in Theorem 2.7, we recover Theorem 3.1 of [15]. [18].

This paper is a slightly modified version of preprint

\section{Open Problems}

For $k>0, p \in N$ and $q \in(0,1)$, let $\psi_{p}(t), \psi_{q}(t)$, $\psi_{(p, q)}(t)$ and $\psi_{(q, k)}(t)$ be the generalized Psi functions as defined in equations (1) - (5).

Problem 1: Under what conditions will the statements:

$$
\begin{aligned}
& \operatorname{In} p+\operatorname{In}(1-q)+\psi_{q}(t)-\psi_{p}(t) \\
& =\sum_{n=0}^{p} \frac{1}{n+t}+(\operatorname{In} q) \sum_{n=1}^{\infty} \frac{q^{n t}}{1-q^{n}} \leq(\geq) 0
\end{aligned}
$$

be valid?

Problem 2: Under what conditions will the statements:

$$
\begin{aligned}
& -\operatorname{In}[p]_{q}-\frac{\operatorname{In}(1-q)}{k}+\psi_{(p, q)}(t)-\psi_{(q, k)}(t) \\
& =(\operatorname{In} q)\left[\sum_{n=1}^{p} \frac{q^{n t}}{1-q^{n}}-\sum_{n=1}^{\infty} \frac{q^{n k t}}{1-q^{n k}}\right] \leq(\geq) 0
\end{aligned}
$$

be valid?

\section{Competing Interests}

The authors declare that there is no competing interest.

\section{Acknowlegement}

The authors are very grateful to the anonymous reviewers for their useful comments and suggestions which helped in improving the quality of this paper.

\section{References}

[1] Y. C. Chen, T. Mansour and Q. Zou, On the complete monotonicity of quotient of Gamma functions, Math. Ineq. \& Appl. 15:2 (2012), 395-402.

[2] R. Diaz and E. Pariguan, On hypergeometric functions and Pachhammer k-symbol, Divulgaciones Matematicas 15(2)(2007), 179-192.

[3] R. Diaz and C. Teruel, q,k-generalized gamma and beta functions, J. Nonlin. Math. Phys. 12(2005), 118-134. 
[4] T. Mansour, Some inequalities for the q-Gamma Function, J. Ineq. Pure Appl. Math. 9(1)(2008), Art. 18.

[5] T. Mansour and A.Sh. Shabani, Some inequalities for the qdigamma function, J. Ineq. Pure and Appl. Math. 10:1 (2009), Article 12.

[6] T. Mansour and A. Sh. Shabani, Generalization of some inequalities for the $\left(q_{1} \ldots . . q_{s}\right)$-Gamma function, Le Matematiche LXVII (2012), 119-130.

[7] F. Merovci, Power Product Inequalities for the $\Gamma_{k}$ Function, Int. Journal of Math. Analysis, 4(21)(2010), 1007-1012.

[8] V. Krasniqi and F. Merovci, Some Completely Monotonic Properties for the (p,q)-Gamma Function, Mathematica Balkanica, New Series 26(2012), 1-2.

[9] V. Krasniqi, T. Mansour and A. Sh. Shabani, Some Monotonicity Properties and Inequalities for $\Gamma$ and $\zeta$ Functions, Mathematical Communications 15(2) (2010), 365-376.

[10] V. Krasniqi, T. Mansour, and A. Sh. Shabani, Some inequalities for q-polygamma function and zeta q-Riemann zeta functions, Ann. Math. Informaticae, 37 (2010), 95-100.

[11] K. Nantomah and M. M. Iddrisu, Some Inequalities Involving the Ratio of Gamma Functions, Int. Journal of Math. Analysis 8(12)(2014), 555-560.
[12] K. Nantomah, M. M. Iddrisu and E. Prempeh, Generalization of Some Inequalities for theRatio of Gamma Functions, Int. Journal of Math. Analysis, 8(18)(2014), 895-900.

[13] K. Nantomah and E. Prempeh, Generalizations of Some Inequalities for the p-Gamma, q-Gamma and k-Gamma Functions, Electron. J. Math. Anal. Appl. 3(1)(2015),158-163.

[14] K. Nantomah and E. Prempeh, Some Sharp Inequalities for the Ratio of Gamma Functions, Math. Aeterna, 4(5)(2014), 501-507.

[15] K. Nantomah and E. Prempeh, Generalizations of Some Sharp Inequalities for the Ratio of Gamma Functions, Math. Aeterna, 4(5)(2014), 539-544.

[16] K. Nantomah, On Certain Inequalities Concerning the Classical Euler's Gamma Function, Advances in Inequalities and Applications, Vol. 2014 (2014) Article ID 42.

[17] K. Nantomah, Generalized Inequalities Related to the Classical Euler's Gamma Function, Turkish Journal of Analysis and Number Theory, 2(6)(2014), 226-229.

[18] K. Nantomah, Further Inequalities Associated with the Classical Gamma Function, arXiv:1506.07393v1. 